\title{
Determination of the Degree of Branching of Comb-Shaped Branched Polystyrenes by a GPC/LS Method*
}

\author{
Tadaya KATO, ${ }^{* * 1}$ Akira KANDA, ${ }^{* 1}$ Akira TAKAHASHI, ${ }^{* 1}$ \\ Ichiro NODA, ${ }^{* 2}$ Shigeaki MAKI, ${ }^{* 2}$ and Mitsuru NAGASAWA ${ }^{* 2}$ \\ ${ }^{* 1}$ Chemistry Department of Industry and Resources, Faculty of Engineering, Mie University, \\ Tsu 514, Japan. \\ ${ }^{* 2}$ Department of Synthetic Chemistry, Faculty of Engineering, Nagoya University, \\ Chikusa-ku, Nagoya 464, Japan.
}

\begin{abstract}
(Received February 15, 1979)
ABSTRACT: A laser light scattering detector with a scattering angle of $90^{\circ}$ is attached to a conventional GPC. The reliability of the instrumentation (GPC/LS method) is tested by measuring the molecular-weight distributions of the standard linear polymers with narrow molecular-weight distribution. The applicability of the GPC/LS method for determining the degree of branching is studied by using comb-shaped branched polystyrenes previously characterized. It is concluded that the GPC/LS method is useful for determining the degree of branching if the type of branching is known and also if the degree of branching is not too high.
\end{abstract}

KEY WORDS GPC / Light Scattering Method / Weight-Average Molecular Weight / Molecular-Weight Distribution / Comb-Shaped Branched Polystyrenes / Degree of Branching /

Several investigators ${ }^{1-4}$ adopted a light scattering detector (LS) as an additional GPC detector and determined the absolute value of the weight-average molecular weight. Since the retention volume $V_{\mathrm{r}}$ of GPC gives the radius of gyration of a polymer, ${ }^{5}$ the combination of the GPC and the LS detector should provide information on the configuration of the polymer, such as its branching. If the type of branching of the polymer is known, we would be able to calculate the degree of branching of the polymer. That is, if the branched samples are comb-shaped polymers as used in the present work, the ratio of the unperturbed square radius of gyration of the branched polymer $\left\langle S^{2}\right\rangle_{\mathrm{ob}}$ to that of the corresponding linear one $\left\langle S^{2}\right\rangle_{\text {ol }}$ of the same molecular weight, $g_{\mathrm{s}}$, is given as a function of the degree of branching $f$, such as, ${ }^{6,7}$

$$
\begin{aligned}
g_{\mathrm{s}} \equiv & \left\langle S^{2}\right\rangle_{\mathrm{ob}} /\left\langle S^{2}\right\rangle_{\mathrm{ol}} \\
= & (1+f \gamma)^{-3}\{1+2 f \gamma \\
& \left.+\left(2 f+f^{2}\right) \gamma^{2}+\left(3 f^{2}-2 f\right) \gamma^{3}\right\}
\end{aligned}
$$

* Presented at the 27th Annual Meeting of the Society of Polymer Science, Japan, in Nagoya, May 24, 1978, Code $1 \mathrm{H} 21$.

**1 To whom correspondence should be addressed. where $\gamma$ is the ratio of the molecular weight of a branch $M_{\mathrm{b}}$ to that of the parent polystyrene $M_{0}$. The values of $\left\langle S^{2}\right\rangle$ for both branched and linear polymers can be estimated from their retention volumes; the molecular weight of the branched polymer can be determined by the LS detector. Thus, the degree of branching $f$ may be calculated from eq 1 using such data. In practice, however, there are various problems involved. For example, theoretically, $g_{\mathrm{s}}$ is usually given in theta solvents, whereas GPC should be carried out in good solvents.

Besides determining the degree of branching, LS and GPC combination is useful for finding the molecular-weight distribution of linear polymers when appropriate standard polymers for calibration of the columns are not available. ${ }^{1-3}$

Usually, an LS detector combined with GPC has a cell specially designed for observing scattered light at very low angles. Theoretically speaking, however, it may not be necessary to measure the scattered light at low angles. The LS detection is sensitive enough to determine weak scattered light at wide angles and the particle scattering factor can be calculated theoretically since eluted polymer fractions from GPC have narrow molecular-weight distributions. The $90^{\circ}$-LS detector is not handicapped by the problem of dust in 
the eluate.

The purpose of this work is to examine the method for determining the degree of branching by using comb-shaped polymers having well defined molecular characteristics as well as linear samples having sharp molecular-weight distributions. The instrument used here was constructed in our laboratory with the scattering angle fixed at $90^{\circ}$.

\section{EXPERIMENTAL}

\section{Materials}

The linear poly $(\alpha$-methylstyrene)s used were prepared by the anionic polymerization method. The molecular characteristics were reported in detail in previous papers. ${ }^{89}$ The molecular-weight distributions of the samples are very narrow and the ratios of the weight-average molecular weights to the number-average molecular weights $\left(M_{w} / M_{n}\right)$ are smaller than 1.05. Linear standard polystyrenes were purchased from Pressure Chem. Co. and Toyo Soda MFG Co. ${ }^{10}$ The samples described as $\mu-201$ and $\mu$ 401 were prepared in our laboratory by the anionic polymerization method.

The samples of comb-shaped branched polystyrenes used were prepared by coupling monodisperse polystyryl anions with chloromethylated monodisperse polystyrenes. ${ }^{11}$ The fractionation, characterization, and GPC measurements of the samples were reported in previous papers. ${ }^{5,12}$ Their molecular characteristics are shown in Tables I and II. Reagent-grade toluene (Wako Pure Chemical Industries $\mathrm{Co}$.) was used as the eluting agent without further purification.

\section{Instrumentation}

The GPC instrument was a high-speed liquid chromatograph HLC-801A of Toyo Soda MFG Co. Six TSK GMH6-type 2 feet columns packed with styrene-divinylbenzene porous gel were operated at the column-oven temperature $40^{\circ} \mathrm{C}$ on toluene with a total pressure drop of $40 \mathrm{~kg} \mathrm{~cm}^{-2}$ and a $1.0 \mathrm{ml} \mathrm{\textrm {min } ^ { - 1 }}$ flow rate of the eluent. Theoretical plate numbers of the columns were larger than 5000 plates/ft. The injection loop volume was $1 \mathrm{ml}$ of a $0.05 \mathrm{wt} \%$ polymer concentration.

A laser light scattering apparatus, as shown in Figure 1, was connected to the outlet of the GPC with a stainless steel pipe. The light source of the LS was a He-Ne laser (Tokyo Kodenshi, type 1M 130D) with $2 \mathrm{~mW}$ power (vertically polarized, wave length in vacuum $632.8 \mathrm{~nm})$. Through three pinholes $(\mathrm{PH}$, $\left.D_{1}, D_{2}\right)$, two mirrors $\left(M_{1}\right.$ and $\left.M_{2}\right)$ and a convex lens $(\mathrm{L}, f=10 \mathrm{~cm})$, the laser beam was forcused on the center of measuring cell $(\mathrm{Ce})$ and was absorbed by a Rayleigh-horn (RH). The beam was partially re-

Table I. Molecular characteristics and experimental results of linear polymers

\begin{tabular}{|c|c|c|c|c|c|}
\hline \multirow{2}{*}{ Samples } & \multirow{2}{*}{$\begin{array}{l}\text { Mol wt } \\
M_{w} / 10^{4}\end{array}$} & \multicolumn{3}{|c|}{ Results determined by GPC/LS } & \multirow{2}{*}{$V_{\mathrm{r}}$ (counts) } \\
\hline & & $M_{w} / 10^{4}$ & $M_{n} / 10^{4}$ & $\left(M_{w} / M_{n}\right)$ & \\
\hline \multicolumn{6}{|c|}{ Polystyrenes } \\
\hline PS 448 & 448 & 457 & 452 & 1.01 & 63.3 \\
\hline PS 200 & 200 & 192 & 186 & 1.03 & 66.2 \\
\hline PS 67 & 67 & 66.1 & 63.0 & 1.05 & 73.6 \\
\hline PS 49.8 & 49.8 & 54.1 & 49.3 & 1.10 & 75.2 \\
\hline PS 42.6 & 42.6 & 44.5 & 41.1 & 1.08 & 76.4 \\
\hline PS 20 & 20 & 19.6 & 19.3 & 1.02 & 82.3 \\
\hline$\mu-201$ & 140 & 159 & 154 & 1.03 & 70.8 \\
\hline$\mu-401$ & 1170 & 1135 & 1116 & 1.02 & 60.8 \\
\hline \multicolumn{6}{|c|}{ Poly $(\alpha$-methylstyrene)s } \\
\hline PAS-14 & 749 & 731 & 707 & 1.03 & 63.4 \\
\hline PAS-13 & 330 & \multicolumn{3}{|c|}{ (used as the reference) } & 65.8 \\
\hline PAS-12 & 182 & 202 & 198 & 1.02 & 69.3 \\
\hline PAS-9 & 119 & 122 & 117 & 1.04 & 71.0 \\
\hline PAS-6 & 44.4 & 46.7 & 43.0 & 1.09 & 77.8 \\
\hline PAS-5 & 34.2 & 30.8 & 30.3 & 1.02 & 79.4 \\
\hline PAS-4 & 20.4 & 18.9 & 18.6 & 1.02 & 82.3 \\
\hline PAS-3 & 14.2 & 13.2 & 12.9 & 1.02 & 85.2 \\
\hline
\end{tabular}


Determination of Degree of Branching by GPC/LS

Table II. Molecular characteristics and experimental results of comb-like branched polystryrenes

\begin{tabular}{|c|c|c|c|c|c|c|}
\hline Samples & $\begin{array}{c}\text { Mol wt of } \\
\text { parent } \\
\text { polymers, } \\
M_{\mathrm{o}} / 10^{4}\end{array}$ & $\begin{array}{c}\text { Mol wt of } \\
\text { a branch, } \\
M_{\mathrm{b}} / 10^{4}\end{array}$ & $\begin{array}{c}\text { Number } \\
\text { of } \\
\text { branching, } \\
f\end{array}$ & $\left(M_{w}\right)_{\mathrm{LS}} / 10^{6}$ & $\begin{array}{c}\text { Results } \\
\text { determined } \\
\text { by GPC/LS, } \\
M_{w} / 10^{6}\end{array}$ & $\begin{array}{c}V_{\mathrm{r}} \\
\text { (counts) }\end{array}$ \\
\hline $\mathrm{J}-2$ & \multirow{3}{*}{9.76} & \multirow{3}{*}{35.8} & 9.3 & 3.41 & 5.24 & 67.2 \\
\hline 5 & & & 4.6 & 1.75 & 1.90 & 70.0 \\
\hline 8 & & & 2.7 & 0.96 & 0.98 & 73.3 \\
\hline $\mathrm{H}-4$ & \multirow{3}{*}{9.76} & \multirow{3}{*}{12.9} & 26.6 & 3.53 & 5.30 & 68.4 \\
\hline 5 & & & 22.6 & 3.01 & 5.06 & 69.5 \\
\hline 6 & & & 18.8 & 2.43 & 4.43 & 69.9 \\
\hline I-4 & \multirow{3}{*}{9.76} & \multirow{3}{*}{17.6} & 23.0 & 4.15 & 4.75 & 68.6 \\
\hline 7 & & & 17.8 & 3.24 & 4.73 & 69.7 \\
\hline 9 & & & 9.9 & 1.84 & 2.73 & 71.5 \\
\hline K-1 & \multirow{5}{*}{8.83} & \multirow{5}{*}{14.7} & 103 & 16.1 & 15.5 & 65.3 \\
\hline 2 & & & 88 & 13.9 & 11.6 & 65.0 \\
\hline 3 & & & 56 & 9.18 & 9.80 & 65.4 \\
\hline 4 & & & 42 & 7.06 & 9.53 & 64.6 \\
\hline 5 & & & 33 & 5.88 & 8.00 & 65.9 \\
\hline L-1 & \multirow{5}{*}{3.68} & \multirow{5}{*}{6.32} & 192 & 12.5 & 12.8 & 65.9 \\
\hline 2 & & & 149 & 9.80 & 9.72 & 66.0 \\
\hline 3 & & & 132 & 8.69 & 9.29 & 67.0 \\
\hline 4 & & & 117 & 7.75 & 7.18 & 67.8 \\
\hline 5 & & & 87 & 5.88 & 7.21 & 66.0 \\
\hline
\end{tabular}

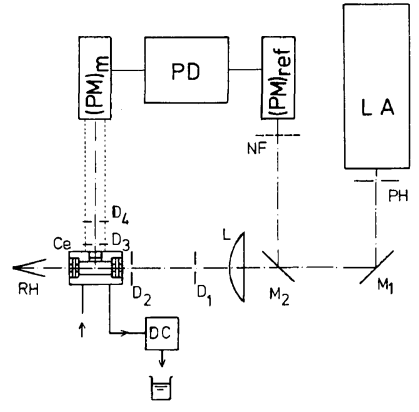

Figure 1. The brief diagram of LS instrumentation. The abbreviations used in the figure are as follows: $\mathrm{Ce}$, light scattering cell; DC, drop counter; LA, He-Ne laser; PD, photoncounting device.

flected by the half mirror $\left(\mathrm{M}_{2}\right)$ and radiated on the reference photomultiplier $(\mathrm{PM})_{\text {ref }}$ (Hamamatsu $\mathrm{TV}$, type R497). A neutral filter (NF) reduced the reference light power by about $10^{-2}$. The measuring cell was made of a glass cylinder with a $2 \mathrm{~mm}$ inner diameter, a $10 \mathrm{~mm}$ length and a $0.032 \mathrm{ml}$ volume. The intensity of the scattering light at a scattering angle of $90^{\circ}$ was measured by the photoncounting method with photomultiplier $(\mathrm{PM})_{\mathrm{m}}$ (Hammamatsu $\mathrm{TV}$, type $\mathrm{R} 464)$ through two pinholes $\left(\mathrm{D}_{3}, \mathrm{D}_{4}\right)$ and an optical guide of glass-fiber. The signal/noise ratios of two detectors were maximized by controlling the attached discriminator and high voltage of the photomultiplier. The counting numbers, $I_{90}$, were digitaled for every $0.1 \mathrm{ml}$.

\section{Data Processing Method}

The relative intensity of the scattered light at the scattering angle $\theta$ is given as follows.

$$
\begin{gathered}
\frac{K c}{\Delta R_{\theta}}=\frac{1}{M_{w} P(\theta)}+2 A_{2} c+\cdots \\
K=\frac{4 \pi^{2} n_{0}^{2}}{\lambda_{0}^{4} N_{\mathrm{A}}}\left(\frac{\partial n}{\partial c}\right)^{2}
\end{gathered}
$$

where $\Delta R_{\theta}$ is the difference between the Rayleigh 
ratio of solution and solvent, $n_{0}$ is the refractive index of solvent, $\lambda_{0}$ is the wave length in vacuum, $N_{\mathrm{A}}$ is Avogadro's number, $c$ is the polymer concentration $\left(\mathrm{g} \mathrm{ml}^{-1}\right), M_{w}$ is the weight-average molecular weight of solute polymer, $A_{2}$ is the second virial coefficient, $(\partial n / \partial c)$ is the refractive index increment and $P(\theta)$ is the particle scattering factor. Since the polymer concentration eluted from GPC column is very low (lower than $10^{-5} \mathrm{~g} \mathrm{ml}^{-1}$ ), the second term of the right side of eq 2 is negligible and thus we have

$$
\Delta R_{\theta}=K c M_{w} P(\theta)
$$

The $P(\theta)$ cannot be neglected at $90^{\circ}$. The intensity of the light was adjusted so that $I_{90}$ was proportional to the Rayleigh ratio $R_{90}$. Thus, the difference between the photoncounting numbers for the solution and solvent, $\Delta I_{90}$, is

$$
\Delta I_{90}=\Psi \Delta R_{90}
$$

where $\Psi$ is the instrumental constant and $\Delta R_{90}$ is the difference between the Rayleigh ratio of the solution and solvent. The $P(90)$ values for linear standard polymers can well be estimated from the theory of Debye, ${ }^{13}$ even in good solvent. ${ }^{8,10}$

$$
\begin{gathered}
P(\theta)=\left(2 / x^{2}\right)\left(\mathrm{e}^{-x}-1+x\right) \\
x=16 \pi^{2}\left(n / \lambda_{0}\right)^{2}\left\langle S^{2}\right\rangle_{z} \sin ^{2}(\theta / 2)
\end{gathered}
$$

where $\left\langle S^{2}\right\rangle_{z}$ is the $z$-average square radius of gyration.

The $P(\theta)$ for comb-shaped branched polymers in theta solvents was calculated by Casassa and Berry. ${ }^{6}$ However, it was previously found that $\left\langle S^{2}\right\rangle_{z}$ in good solvents cannot be determined by applying the $P(\theta)$ of Casassa and Berry but can more reasonably be determined by using eq 6 of Debye. ${ }^{5}$ This may be because the branches of our comb-shaped polymers are so long that their shapes may be approximated as star-shaped polymers. ${ }^{12}$ In this work, we employed eq 6 for calculating $P(90)$ for our comb-shaped branched polymers. Thus, the weight-average molecular weight is given by

$$
M_{w}=\frac{\sum_{i} c_{i} M_{i}}{\sum_{i} M_{i}}=\frac{1}{\Psi K P(90)} \frac{\sum_{i}\left(\Delta I_{90}\right)_{i}}{\sum_{i} c_{i}}
$$

where $\sum_{i}\left(\Delta I_{90}\right)_{i}$ is the total peak area of the LS detector and $\sum_{i} c_{i}$ is the total peak area of the differential refractometer (DRI). The number- average molecular weight is also expressed by

$$
M_{n}=\frac{\sum_{i} c_{i}}{\sum_{i}\left(c_{i} / M_{i}\right)}=\frac{\sum_{i} c_{i}}{\Psi K P(90) \sum_{i}\left\{c_{i}{ }^{2} /\left(\Delta I_{90}\right)_{i}\right\}}
$$

For highly branched polymers, however, eq 9 cannot be used, as will be explained latter. The instrumental constant $\Psi$ was calculated from the data with the poly $\left(\alpha\right.$-methylstyrene) PAS-13 sample $\left(M_{w}=3.30\right.$ $\left.\times 10^{6}\right)$.

\section{RESULTS AND DISCUSSION}

In Figure 2, the LS chromatograms for several $\operatorname{poly}(\alpha$-methylstyrene) solutions at equal concentrations $(0.05 \mathrm{wt} \%)$ are shown, in comparison with those of the DRI detector. DRI shows chromatograms of almost equal areas for all samples, whereas the peak area of LS chromatograms increases with molecular weight of polymer. Examples of combshaped branched polystyrene are also shown in Figure 3. The calculated values of $M_{w}$ and $M_{n}$ from the chromatograms of GPC/LS sysrems are shown in Tables I and II. The last columns in the tables represent the retention volumes corresponding to the maximum peak positions.

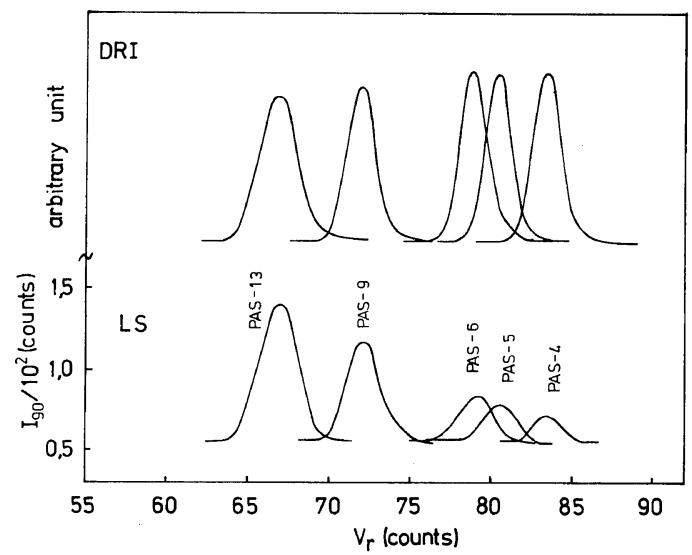

Figure 2. Examples of chromatograms of $\operatorname{poly}(\alpha-$ methylstyrene)s obtained by laser light scattering measurement, compared with those of DRI detector.

\section{Molecular-Weight Determination by the GPC/LS Method}

Figure 4 shows the comparison of $\left(M_{w}\right)_{\mathrm{GPC} / \mathrm{LS}}$ observed by GPC/LS method in this work with $\left(M_{w}\right)_{\text {LS }}$ determined by conventional light scattering 


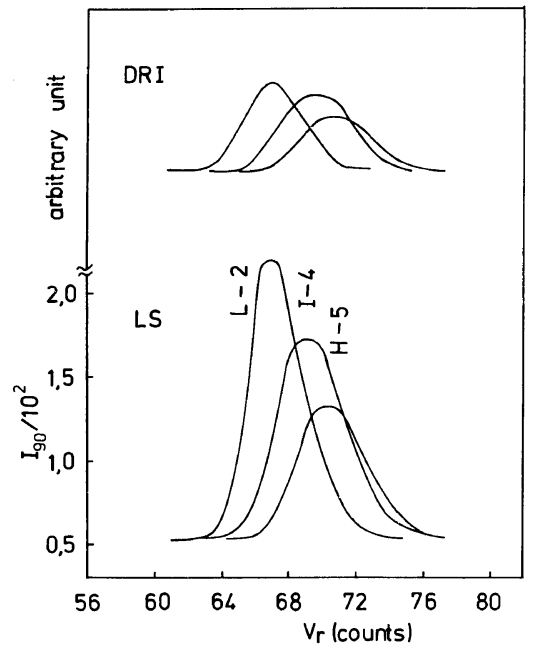

Figure 3. Examples of the chromatograms of combshaped branched polystyrenes.

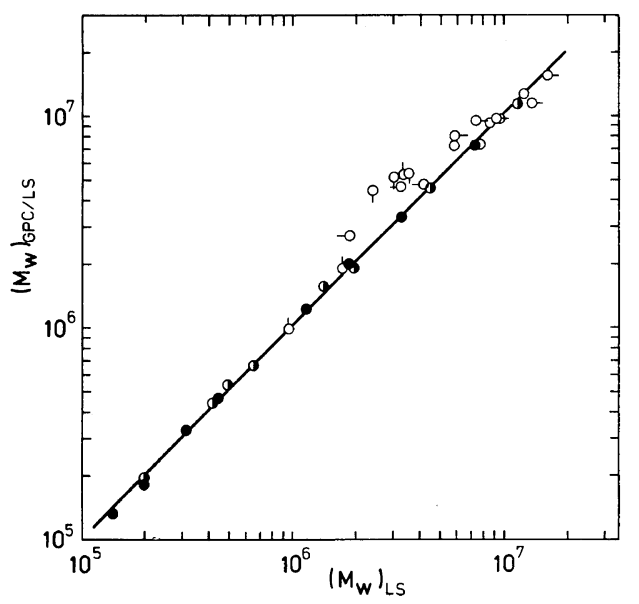

Figure 4. The comarison between the $\left(M_{w}\right)_{\mathrm{GPC} / \mathrm{LS}}$ and the $\left(M_{w}\right)_{\mathrm{LS}}$. The notations denote as follows: $\mathbf{D}, \operatorname{poly}(\alpha-$ methylstyrene) samples;, $\mathrm{H} ; \mathrm{Q}, \mathrm{I} ;-\mathrm{O}, \mathrm{J} ; \mathrm{O}, \mathrm{K} ; \mathrm{O}, \mathrm{L}-$ series of comb-shaped branched polystyrenes, respectively.

instrument (LS) used in previous papers. ${ }^{5,8-12}$ For linear polymers the agreement between $\left(M_{w}\right)_{\mathrm{GPC} / \mathrm{LS}}$ and $\left(M_{w}\right)_{L S}$ is excellent. Moreover, except at both tails of the chromatographic peak where the determination of polymer concentration by DRI becomes errorneous, $\left(M_{w}\right)_{\mathrm{GPC} / \mathrm{LS}}$ is the same throughout the entire peak, as expected from the narrow molecular-weight distributions of the samples. Examples of the molecular weights of fractions thus determined are shown in Figure 5. The molecular

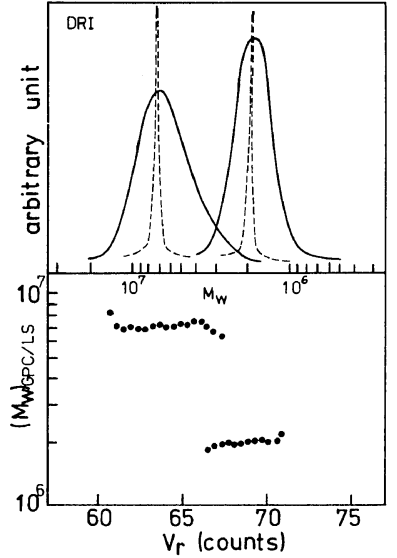

Figure 5. Examples of the molecular weight distribution determined by GPC/LS method throughout the peak width of chromatograms. Samples: PAS-14 (left) and PAS-12 (right).

weight distributions of the samples constructed with the GPC chromatogram and the molecular-weights determined directly by GPC/LS method are also shown by solid and broken lines respectively in Figure 5. It is clear that the chromatograms in Figures 2 and 5 are due to the broadening effect of the columns. The values of $\left(M_{w} / M_{n}\right)_{\mathrm{GPC} / \mathrm{LS}}$ are also shown in Table I. These values are reasonably narrow in comparison with those reported previously ${ }^{8,10}$

For some samples of comb-shaped branched polystyrenes, $\left(M_{w}\right)_{\mathrm{GPC} / \mathrm{LS}}$ is slightly larger than $\left(M_{w}\right)_{L S}$, as shown in Table II and in Figure 4. The reason for the disagreement between $\left(M_{w}\right)_{\mathrm{GPC} / \mathrm{LS}}$ and $\left(M_{w}\right)_{\mathrm{LS}}$ is not clear. $\left(M_{w} / M_{n}\right)_{\mathrm{GPC} / \mathrm{LS}}$ cannot be determined by this method since the molecular size of comb-shaped polymers does not increase with a greater degree of branching. That is, an apparent resolution limit is observed in the $V_{\mathrm{r}}$-number of the branching $(f)$ curve. If the polymers are rather highly branched, their retention volumes are observed near the resolution limit, as shown in Table II. ${ }^{14}$

\section{Determination of the Degree of Branching}

It was reported in a previous paper ${ }^{5}$ that $V_{\mathrm{r}}$ of the GPC is determined by the radius of gyration $\left\langle S^{2}\right\rangle$. Therefore, we can determine $\left\langle S^{2}\right\rangle$ of comb-shaped polymers from $V_{\mathrm{r}}$ by using the relationship between $\left\langle S^{2}\right\rangle$ and $V_{\mathrm{r}}$ for linear polymers and calculate $g_{\mathrm{s}} \equiv\left\langle S^{2}\right\rangle_{\mathrm{b}} /\left\langle S^{2}\right\rangle_{1}$ at the same molecular weight in good solvents. Then, we can estimate the average 
degree of branching $f$ if we know the type of branching of the polymer as well as $M_{0}$ and $M_{\mathrm{b}}$.

However, there are a few problems involved in this method for determining the degree of branching: (1) theoretically and experimentally, $g_{\mathrm{s}}$ of comb-shaped polymers degreases with increasing degree of branching but levels off at higher degrees of branching. Therefore, the degree of branching of combshaped polymers can be determined only if $f$ is low. Among the present samples, series J may be used for testing this method. (2) GPC is generally carried out in good solvents. The theory for $g_{\mathrm{s}}$ in theta solvents cannot be used. Moreover, (3) it was reported that the theoretical $g_{\mathrm{s}}$ as a function of $f$ and $\gamma$ does not agree with experimental data even at the theta condition for linear polymers. ${ }^{12}$ Since we have the experimental relationship between $g_{\mathrm{s}}$ and $f$ in the same solvent as used in the present experiments, which is similar to Figure 7 in reference 12, we may tentatively use the experimental graph for estimating $f$ from $g_{\mathrm{s}}$. The degree of branching thus estimated is shown in Table III in comparison with $f$ determined previously. The big disagreement found for sample J2 arises from the disagreement between $\left(M_{w}\right)_{\mathrm{GPC} / \mathrm{LS}}$ and $\left(M_{w}\right)_{\mathrm{LS}}$ in Table II. If we employ $\left(M_{w}\right)_{\mathrm{LS}}$ $\left(3.41 \times 10^{6}\right)$ instead of $\left(M_{w}\right)_{\mathrm{GPC} / \mathrm{LS}}\left(5.24 \times 10^{6}\right)$ to deduce $\left\langle S^{2}\right\rangle_{1}$, we have $f=9$. In Table III, the values of $f$ calculated from eq 1 using $\left(M_{w}\right)_{\mathrm{GPC} / \mathrm{LS}}$ are also shown for reference.

Table III. The numbers of branching observed by GPC/LS method

\begin{tabular}{cccc}
\hline \multirow{4}{*}{$\begin{array}{c}f \text { calculated } \\
\text { by } M_{\mathrm{o}} \\
\text { and } M_{\mathrm{b}}\end{array}$} & $\begin{array}{c}f \text { calculated by GPC/LS method } \\
\text { using the } \\
\text { experimental } \\
g_{\mathrm{s}}-f \\
\text { relationship }\end{array}$ \\
\hline from eq 1 \\
$\mathrm{~J}-2$ & 9.3 & $35 \quad(9)^{\mathrm{a}}$ & 12 \\
$\mathrm{~J}-5$ & 4.6 & 6 & 5 \\
\hline
\end{tabular}

${ }^{\text {a }} \mathrm{M}=3.41 \times 10^{6}$ is employed.

If the degree of branching is not so high that the retention volume of the comb-shaped polymer is below the resolution limit in $V_{\mathrm{r}}-f$ curve, we can determine the distribution of the degree of branching in the sample. The experimental results for $\mathrm{J}-5$ and $\mathrm{J}$ 8 are shown in Figure 6. The degrees of branching of

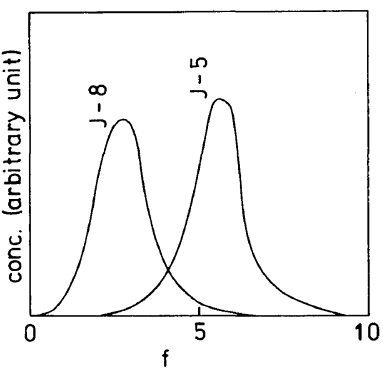

Figure 6. Examples of the distribution of degree of branching by GPC/LS method.

the other samples are so high that their retention volumes are near the resolution limit on the $V_{\mathrm{r}}-f$ curve and their chromatograms are determined by the broadening effect of the columns.

In conclusion, the GPC/LS method is useful for determining molecular-weight distributions of linear polymers. For this purpose it is not necessary to use a low angle LS detector. To determine the degree of branching, it is advisable to use a low angle LS detector (about $10^{\circ}$ ) since $P(\theta)$ for branched polymers in good solvents is not well understood. The use of the theory of Debye for estimating $P(\theta)$ for branched polymers is tentatively recommended when we employe a $90^{\circ}$-LS detector. Even if we can determine the molecular weights of fractions correctly, we cannot determine the degree of branching with complete certainty since the theory of $g_{\mathrm{s}}$ in good solvents has not yet been given. The values of $f$, based on certain assumptions concerning $P(\theta)$ and $g_{\mathrm{s}}$, are however, fairly reasonable if the degree of branching is not high.

Acknowledgment. We wish to thank Dr. Y. Kato of Toyo Soda MFG Co. for his kind supply of GPC columns and Prof. H. Matsuura of Kanazawa University for his help in making the photoncounting device.

\section{REFERENCES}

1. A. C. Ouano and W. Kaye, J. Polym. Sci., A, 12, 1151 (1974).

2. A. C. Ouano, J. Chromatogr., 118, 303 (1976).

3. A. C. Ouano, J. Colloid and Interface Sci., 63, 275 (1978).

4. G. Meyerhoff, Separation Sci., 6, 239 (1971).

5. T. Kato, A. Itsubo, Y. Yamamoto, T. Fujimoto, and M. Nagasawa, Polym. J., 7, 123 (1975).

6. E. F. Casassa and G. C. Berry, J. Polym. Sci., A-2, 4, 
881 (1966); E. F. Casassa and Y. Tagami, J. Polym. Sco., A-2, 6, 63 (1968).

7. G. C. Berry, J. Polym. Sci., A-2, 6, 1551 (1968).

8. T. Kato, K. Miyaso, I. Noda, T. Fujimoto, and M. Nagasawa, Macromolecules, 3, 777 (1970).

9. I. Noda, K. Mizutani, T. Kato, T. Fujimoto, and M. Nagasawa, Macromolecules, 3, 787 (1970).
10. M. Fukuda, M. Fukutomi, Y. Kato, and T. Hashimoto, J. Polym. Sci., 12, 871 (1974).

11. T. Fujimoto, H. Narukawa, and M. Nagasawa, Macromolecules, 3, 57 (1970).

12. I. Noda, T. Horikawa, T. Kato, T. Fujimoto, and M. Nagasawa, Macromolecules, 3, 795 (1970).

13. P. Debye, J. Phys. Colloid Chem., 51, 18 (1947).

14. T. Kotaka, J. Appl. Polym. Sci., 21, 501 (1977). 